\title{
Association of sleep duration and weekend catch-up sleep with suicidal ideation among adolescents with asthma: secondary data analysis based on a nationwide cross-sectional study
}

\author{
Seung Chan Jeong ${ }^{1}$, Chang Woon Kim¹, Sang Won Hwang ${ }^{1}$, Seon Hui Jo ${ }^{1}$, and Sung Hoon \\ $\mathrm{Kim}^{1}$ \\ ${ }^{1}$ Samsung Changwon Hospital
}

June 17, 2020

\begin{abstract}
Objective: Little is known regarding the effect of sleep duration and weekend catch-up sleep (WCUS), sleep time on weekends that exceeds sleep time on weekdays, on suicidal ideation among adolescents with asthma. We explored whether sleep factors were associated with suicidal ideation among adolescents with asthma. Hypothesis: Adolescents with asthma are likely to have shorter sleep duration and longer WCUS than those without asthma. Study Design: Secondary data analysis for cross-sectional, self-administered, online survey. Patients Selection: Adolescents participating in the annual Korean Youth Risk Behavior Webbased Surveys (KYRBS, completed from 2013 to 2017). Methodology: The KYRBS data were obtained from a stratified, multistage, clustered sample. Students responded prior diagnoses of asthma and sleep time. Associated factors for suicidal ideation were tested by logistic regression models. Results: Among 34,067 and 363,003 adolescents with and without asthma, respectively, adolescents with asthma had poorer sleep satisfaction (44.6\% vs. $42.6 \%$ ), slept less (sleep duration $6.59 \pm 0.94$ vs $7.29 \pm 1.45 \mathrm{hr}$ ), and had longer WCUS $(3.13 \pm 0.01 \mathrm{vs} 2.29 \pm 0.01 \mathrm{hr})$ than did those without asthma. The odds ratio (OR) of suicidal ideation in those with short sleep duration (defined by [?]5h) was 1.37 (95\% Confidence Interval [CI], 1.301.43). Notably, long WCUS ([?]2hr) was significantly associated with a decreased risk of suicidal ideation (OR, 0.88 [95\% CI, 0.86-0.93]). Conclusion: Although further research is needed to clarify this association, long WCUS, which is a compensatory phenomenon for insufficient weekday sleep, protects against suicidal ideation in adolescents with asthma.
\end{abstract}

\section{KEYWORDS}

adolescent, asthma, sleep duration, suicidal ideation, weekend catch-up sleep

\section{1 | INTRODUCTION}

Although the prevalence of asthma has started to plateau and even decline in adolescents in Western countries, ${ }^{1}$ asthma remains one of the most common chronic pediatric diseases with a significant impact on quality of life (QoL). ${ }^{2}$ One of the ways that asthma affects quality of life is through its impact on sleep, including more frequent nighttime awakenings due to night asthma attacks and sleep-disordered breathing. ${ }^{3-7}$ Nocturnal asthma is a common complication, with [?] $90 \%$ of the episodes of dyspnea in asthmatics occurring at night. ${ }^{8}$ These nocturnal asthma symptoms typically occur between 04:00 and 06:00 h. ${ }^{8}$ The most studied causes and contributing factors to asthma exacerbations at night include night-time physiological changes (e.g., accidental coughing, wheezing, and breathing difficulties), ${ }^{6,7,9}$ and circadian changes in ventilation, airway responsiveness and inflammation, mucociliary clearance, ventilatory responses to hypercapnia and hypoxia, and hormone levels. ${ }^{6}$ Eventually, nocturnal symptoms trigger awakening during sleep, which impairs daily activities, increases morbidity, induces psychometric changes, reduces QoL and these changes can even contribute to suicidality. ${ }^{10}$ However, the association between sleep duration and asthma is conflicting. 
Some studies have found that children with asthma have generally shorter sleep durations than do those without asthma, ${ }^{11}$ while other studies have not found such differences in adolescents. ${ }^{12}$ Another report found that sleep duration in asthmatics is significantly longer than it is in non-asthmatics. ${ }^{13}$ Recently, compared with $7-8 \mathrm{~h}$ of sleep per night in adolescents, both short sleep duration (sleeping for $<7 \mathrm{~h}$ ) and long sleep duration (sleeping for [?]9 h) had $1.22(95 \%$ CI 1.07, 1.40) and $1.31(1.06,1.63)$ times higher OR of asthma, respectively. ${ }^{7}$

Weekend catch-up sleep (WCUS) is defined as sleep time during the weekend that exceeds sleep time on weekdays. ${ }^{5,14}$ WCUS is not only an indicator of insufficient weekday sleep, but also a compensatory phenomenon for weekday sleep debt. ${ }^{5,14}$ Adolescents tend to sleep for shorter durations during the night and exhibit a delayed sleep phase than do children. ${ }^{15}$ Adolescents also tend to sleep less than children do during school days because of the pressure of school work. In order to compensate for insufficient weekday sleep over the entire week, adolescents then tend to sleep more on the weekends. ${ }^{9,15}$ The effects of WCUS on one's overall health are conflicting. Some studies have found that WCUS is associated with a lower risk of hypertension, ${ }^{14}$ obesity prevention in adults, ${ }^{16}$ and lower frequency of asthma in adolescents. ${ }^{5}$ In contrast, WCUS had adverse effects on executive functions, ${ }^{17}$ and contributes to suicidal attempts and self-injury in children and adolescents. ${ }^{18}$ Sleep disturbances in asthma are well known. However, to the best of our knowledge, no prior studies have addressed the influence that WCUS has on suicidal ideation in adolescents with asthma. Current suicidal ideation is considered one of the most important clinical predictors of future attempts and death by suicide. ${ }^{19}$ Therefore, it is crucial to identify the sleep factors that are associated with suicidal ideation in adolescents with asthma in order to reduce suicide in this group.

Given this background, we hypothesized that adolescents with asthma are more likely to have shorter total and weekday sleep duration than are peers without asthma. We also suspected that adolescents with asthma would have longer weekend sleep duration and longer WCUS to compensate for their school day sleep deprivation than would adolescents without asthma. This study had two aims. First, we investigated and compared the differences in the clinical characteristics, sleep pattern, and mental health status in adolescents with and without asthma. Second, we identified the associated sleep factors for suicidal ideation in adolescents with asthma. This second step was the main purpose of this study. Therefore, this study was conducted to investigate the association between sleep duration and WCUS with suicidal ideation in adolescents with asthma in a nationally representative Korean dataset.

\section{2 | MATERIALS AND METHODS}

\subsection{Study design and participants}

This study was designed as a secondary data analysis using and interpreting data drawn from the third through thirteenth Korean Youth Risk Behavior Web-based Surveys (KYRBSs) completed across 7 years from 2013 to 2019. KYRBS was initiated by the Korean Ministry of Education, Ministry of Health and Welfare, and Centers for Disease Control and Prevention (KCDC) in 2005. The KYRBS is a cross-sectional, self-administered, anonymous, online survey that is conducted annually in a nationally representative sample of Korean adolescents aged 12-18 years. The Institutional Review Board (IRB) of the KCDC approved this online statistical research during the entire survey every year. In order to obtain a nationally representative sample, the KYRBS used the stratified three stage random cluster sampling method, which included stratification, sample allocation, and stratified cluster sampling, as is described. The study population was stratified by geographic region and school type to minimize sampling error. In sample allocation, 400 middle schools and 400 high schools were selected by proportional sampling to match the study population every year. In stratified cluster sampling, sample classes were selected by simple randomization sampling from the selected schools. All of the participants provided written informed consent (both directly and from their parents or legal guardians) for approval of survey access. Every student participated voluntarily and completed the survey by logging on to the survey's Internet webpage at their school's computer laboratory using a randomly assigned unique identification number. The participants were not able to skip to subsequent questions without providing proper answers to every question. The online system does not accept omitted responses. Data were considered to be missing if there was any logical error or if there was outlier. In the 
present study, we obtained the raw data that are publicly available from the online site of KYRBSs (https://www.cdc.go.kr/yhs/home.jsp). The reasons for drop-out or non-participation were not available. Our study protocol for secondary analysis and interpretation was reviewed and approved by the IRB of Samsung Changwon Hospital (IRB No. 2020-06-009).

\subsection{Dependent variable}

Participants were asked about their history of asthma using the following question: "Have you ever been diagnosed with asthma by a doctor at any point in your life or within the last 12 months?" The accepted responses were either "yes" or "no." The disease severity, current symptoms, presence of management, and treatment modality were not assessed.

\section{3 | Independent variables of main interest: sleep-related factors}

The major independent variable for our study was sleep duration and WCUS. In the KYRBSs, self-reported wake up time and bedtime were determined on the basis of participants' responses to the following questions separately for both weekdays and the weekend: (1) "What time did you usually go to bed and wake up during the weekdays (school days) over the last week?" and (2) "What time did you usually go to bed and wake up during the weekend over the last week?" The responses were provided separately for weekdays and the weekend as follows: (1) sleep time: ( ) o'clock ( ) minute AM/PM and (2) wake up time: ( ) o'clock ( ) minute AM/PM. The responses for sleep time on both weekdays and weekends were categorized as: [?]9:00, 9:00-10:00, 10:00-11:00, 11:00 PM-12:00AM, 12:00-1:00, 1:00-2:00, and [?]2:00 AM. Because wake up time was influenced by school attendance, the responses for wake up time were categorized as [?]5:00, 5-6:00, 6-7:00, 7:00-8:00, and [?]8:00 AM on weekdays, and [?]7:00, 7:00-8:00, 8:00-9:00, 9:00-10:00, 10:00-11:00, and [?]11:00 AM on weekends. We defined the subjects who went to sleep after $2 \mathrm{AM}$ as night owls, and those who woke up before $7 \mathrm{AM}$ as early larks based on the previous literature. ${ }^{5}$ Sleep duration was also separately calculated for weekdays (sleep duration on school days) and weekends (sleep duration on school-free days) on the basis of their previous responses. The average sleep duration was calculated using the following weighted mean value: $(5 \times$ weekday sleep duration $+2 \times$ weekend sleep duration $) \div 7,5,15$ and categorized as: [?] 5 , $5-6,6-7,7-8,8-9$ and [?]9h. Based on the recommendations from the National Sleep Foundation's sleep time duration criteria, ${ }^{20}$ the reference sleep duration was defined by $7-8$ hours per day in this study. WCUS was calculated as the average weekend sleep duration minus average weekday sleep duration, ${ }^{5,15}$ and this was divided into four categories: [?]0 hour, 0-1 hour, 1-2 hours, and [?] 2 hours. Non-CUS has the same meaning as CUS [?] 0 hour. ${ }^{21}$ The levels of sleep satisfaction were assessed by the degree of recovery from fatigue by sleep according to the following question: "How satisfied are you with your sleep during the last week?" We re-categorized sleep quality into the following three groups: enough (plenty and enough), a little, and not enough (not enough and never enough).

\section{4 | Covariate variables}

\section{1) Demographic and socioeconomic variables}

Information on demographic and socioeconomic characteristics (age, sex, school grade [middle school high school], residential type, perceived socioeconomic state [SES], and academic achievements) was evaluated. The type of residence was assessed as living with parents and without parents (such as living with relatives, living independently such as in a dormitory, or in an orphanage). The degree of SES and academic achievements were initially categorized as high, middle-high, middle, middle-low, and low. However, we categorized these as high (high or middle-high), middle (middle), or low (middle-low or low).

\section{2) Health-related behavioral variables}

The frequency of breakfast consumption was assessed with the question, "During the past 7 days, how many days did you eat breakfast?" with allowed responses of "never, $1,2,3,4,5,6$, or 7 days." Those who indicated eating breakfast less than 2 days per week were called "breakfast skippers" according to the standards set in the survey. The frequency of vigorous exercise in the preceding 7 days, which was accompanied by very fast breathing or sweating, and by $>20$ minutes of activity was also assessed. Vigorous exercise was classified 
into none, occasional (1-3 days), and frequent (more than 4 days per week). Participants were considered to be current smokers if they answered "more than 1 day over the past month" to the question "Do you smoke?" For alcohol consumption status, the current drinker was indicated by a reply of "more than 1 day over the past month" to the question 'Do you drink alcohol?" in the standpoint of survey.

\section{3) Emotional variables including suicidality}

Psychological status was assessed using the following four variables: perceived health status, perceived level of happiness, perceived level of stress, and experience of depressive symptoms. Subjective healthiness was assessed with the following question: "What do you think about your health state?" The answer to this question was categorized as follows: healthy, average, and unhealthy. Perceived level of happiness was assessed based on a self-rating happiness scale and classified into happy, average, and unhappy. Perceived stress status was also assessed with the following question: "How often do you feel stress?" and was categorized as follows: often, sometimes and rarely. The following question was used to assess depression: "Within the last year, did you feel sad, blue, or depressed that caused cessation of your usual activities almost every day for two weeks or more?" This question was based on KYRBS and was answered with a binary response (yes or no). The measurement of suicidal ideation was also a binary dependent variable (yes or no) with the examining question being, "During the past 12 months, have you ever seriously thought of committing suicide?" Repetition of depressive mood and suicidal ideation were not assessed.

\section{5 | Statistical analysis}

We conducted all of the statistical analyses using complex sample procedures of the Statistical Package for the Social Sciences (SPSS) software program version 21.0 (IBM Corp., Armonk, NY, USA). Because the KYRBS data were collected through a representative, stratified, and clustered sampling method (as a statistical representative of the general population), data from the survey were weighed based on the sample design. Descriptive statistics were used to depict the basic characteristics of the study population. The chisquare test for categorical variables and independent t-test for continuous variables were used appropriately to compare the general characteristics between subjects with and without asthma. Following the selection of significant covariates using univariable logistic regression analysis, multivariate logistic regression analysis was carried out to determine which sleep-related factors (e.g., sleep satisfaction, sleep duration, and WCUS) were independently contributing to the risk of suicidal ideation in adolescent asthmatics: Model 1 adjusted for sex and grade; Model 2 adjusted for Model 1 variables + socioeconomic variables (residential type, SES, and academic achievement) and health-related behavioral variables (breakfast skipping, smoking, alcohol drinking, and vigorous exercise); and Model 3 adjusted for Model 2 variables + psychological variables (perceived status of health, perceived level of happiness, perceived frequency of stress, and experience of depressive symptoms). The results were expressed using adjusted odds ratios (OR) and 95\% confidence intervals (CI). Statistical significance was indicated by $p$ - values $<0.05$ in all tests.

\section{3 | RESULTS}

\subsection{Demographic characteristics of participants}

During the 7 years, a total of 457,685 among 475,575 targeted adolescents completed the survey with a total response rate of $96.2 \%$ (range 95.3-97.2\%). Among them, 60,615 participants did not provide available sleep records. Therefore, their data was considered to be missing and were excluded. This left a total of 34,067 adolescent with asthma and 363,003 adolescents without asthma. The overall prevalence rate of asthma during the entire survey was $8.7 \%$ (Table 1 ). Table 2 shows the general characteristics of the participants and the differences between subjects with asthma and without. Especially, adolescents with asthma selfreported unhealthier and unhappier perceptions, had more stress, higher levels of depression, and more frequent suicidal ideation and suicidal attempts than did those without asthma.

\section{2 | Sleep parameters among adolescents with and without asthma}

Table 3 shows the differences of sleep parameters between the asthma groups and non-asthma groups. Adolescents with asthma were significantly different from those without asthma in terms of every sleep 
variable. Most of all, sleep satisfaction was significantly poorer (sleep satisfaction not enough: $44.6 \%$ vs. $42.6 \%$ ) in the asthma group than it was in the non-asthma group. Adolescents with asthma slept less than did those without asthma (average sleep duration $6.59+-0.94$ vs $7.29+-1.45 \mathrm{hr}$, proportion of less than $5 \mathrm{hr}$ of sleep duration was $8.6 \%$ vs $7.6 \%$, respectively, $\mathrm{p}<0.001)$. Asthmatics were also more likely to be night owls on both weekdays ( $22.6 \%$ vs $21.2 \%$ ) and weekends $(41.8 \%$ vs $41.2 \%)$, and to be early larks on weekdays $(42.4 \%$ vs $42.0 \%$ ), while less likely to be on weekends ( $4.0 \%$ vs $6.3 \%$ ). The weekday sleep duration $(5.58+-1.81$ vs $6.92+-1.43 \mathrm{hr})$ was shorter, while the weekend sleep duration $(9.11+-2.75$ vs $8.21+-2.91$ hr) was significantly longer in adolescents with asthma than it was in those without. In addition, the WCUS duration was significantly longer in the adolescents with asthma than it was in those without asthma (3.13 +-0.01 vs $2.29+-0.01 \mathrm{hr}$ ). The proportion of longer WCUS among adolescents with asthma was $48.9 \%$, and that among adolescents without asthma was $43.3 \%$.

\section{3 | Associated sleep factors for suicidal ideation of subjects with asthma}

Table 4 lists the adjusted OR for suicidal ideation according to sleep parameters. After adjusting (model 1), the OR of sleep dissatisfaction was 2.47 (95\% CI, 2.39-2.56) times higher risk of suicidal ideation than those with sleep satisfaction. In models 2 and 3, the ORs of sleep dissatisfaction were 2.30 (95\% CI, 2.22-2.38) and 1.21 (95\% CI, 1.16-1.25), respectively. With regard to the average sleep duration, when we defined the sleep reference as $7-8 \mathrm{~h}$, the OR of short sleep ([?]5h) was 1.80 (95\% CI, 1.72-1.87) in model 1, $1.87(95 \%$ CI, 1.79-1.95) in model 2, and 1.37 (95\% CI, 1.30-1.43) in model 3. Notably, long WCUS was significantly associated with a decreased rate of suicidal ideation among adolescents with asthma (OR: 0.84 [95\% CI, 0.82-0.87] in model 1, 0.86 [95\% CI, 0.83-0.88] in model 2, and 0.88 [95\% CI, 0.86-0.93] in model 3). The presence of asthma itself was also significantly associated with suicidal ideation among adolescents with asthma (1.31, 1.30, and 1.09).

\section{4 | DISCUSSION}

The main finding of our study was that sleep dissatisfaction, short sleep, and asthma were all associated with an increased risk of suicidal ideation. However, long WCUS was associated with a decreased risk of suicidal ideation in Korean adolescents with asthma. To the best of our knowledge, this is the first study to investigate the relationship between WCUS and suicidal ideation in adolescents with asthma after adjusting for multiple potential confounding factors.

Our results indicate that short sleep duration is significantly associated with suicidal ideation in adolescents with asthma. Approximately 8-10 hours and 7-9 hours of sleep are considered sufficient for teenagers and young adults, respectively. ${ }^{20}$ In general, adolescents are considered to be chronically sleep deprived with decreased total sleep duration and increased daytime sleepiness. ${ }^{12}$ This chronic sleep deprivation is due to a variety of factors including decreased parental control, inconsistent bedtimes, increased schoolwork, poor sleep hygiene (e.g., caffeine use late in the day, technology in the bedroom, smartphone overuse), and a discordance between biological circadian rhythms and early school start times. ${ }^{12}$ In Korea, most middle- and high-school classes start before 8:30 AM. And many Korean adolescents attend private academic institutions and study late at night even on the weekends. ${ }^{15}$ These students face strong psychosocial pressure to attain sufficient grades for admission into prestigious universities. ${ }^{7,15}$ The link between short sleep duration and suicidal ideation in adolescents with asthma is complex and not fully understood. However, there are several possible explanations for this association.

First, sleep deprivation itself is considered to be a stress-inducing factor for adolescents with a higher risk of several mental disorders such as behavioral disorders, ${ }^{22}$ substance abuse, ${ }^{22}$ depression,${ }^{22,23}$ and suicidality. ${ }^{22}$ In adolescents with asthma who have sleep problems commonly, including prolonged sleep onset latency, difficulty in falling asleep, frequent nocturnal awakenings, daytime sleepiness, and early awakenings, ${ }^{24}$ even when their asthma symptoms are well controlled, ${ }^{12}$ short sleep duration may be associated with higher risk of suicidal ideation

In addition, given its long-term disease course, asthma is associated with depression due to the need for chronic treatment and the repeated exacerbations that may themselves cause hopelessness. ${ }^{25}$ The chronic 
nature of this disease may lead to increased asthma symptoms through risk-taking behaviors (smoking, drinking, non-adherence to medication and poor illness control in general $)^{25}$ and consequently increased suicide risk. ${ }^{26,27}$ As it is known that people with asthma have a significantly increased risk of suicidal ideation, ${ }^{28}$ suicidal ideation may become more prevalent in adolescents with asthma when they become sleep deprived.

Third, in patients with asthma, the prevalence of obstructive sleep apnea (OSA) and sleep disordered breathing (SBD) is consistently higher than it is in the population without asthma. ${ }^{24,29,30}$ Asthma seems to be a consistent and independent risk factor for SDB $\left(\mathrm{OR}=1.9\right.$ [1.69-2.18]). ${ }^{29}$ Gutierrez et al. found that maximal percentage of rapid eye movement (REM) $\mathrm{SpO}_{2}$ desaturation, and the REM obstructive apnea-hypopnea in$\operatorname{dex}(\mathrm{OAHI})$ were significantly higher in asthmatic children with OSA than they were in subjects with OSA alone. ${ }^{31}$ These findings may result in sleep deprivation in adolescents with asthma, and then may be associated with a high risk of suicidal ideation related to the aforementioned negative mental health state in asthma.

Another notable finding in our study was that adolescents with asthma were more likely to be night owls on both weekdays and weekends, while be early larks only on weekdays. As we hypothesized, adolescents with asthma had shorter weekday sleep duration and longer weekend sleep duration than did those without asthma. These findings may be explained by the sleep characteristics and problems of asthma itself, including nocturnal symptoms and the aforementioned educational uniqueness in Korea. These differences may also be related to the longer WCUS in adolescents with asthma than that of those without asthma for sleep debt compensation. Because individual sleep needs vary, WCUS is not only an indicator of the degree of insufficient weekday sleep, ${ }^{5}$ but also is a compensatory behavior to cope with weekday sleep debt. ${ }^{32}$ A recent study found that the risk for anxiety and depression was higher among adults who did not have WCUS than it was among those who did. ${ }^{32}$ Long WCUS was associated with a decreased frequency of asthma and depression in Korean adolescents. ${ }^{5,15}$ However, it is unclear whether WCUS has advantageous effects on emotional states of adolescents with asthma, because sleep hygiene guidelines recommend regularizing sleep time and wake-up time ${ }^{15,20}$ In particular, the association between suicidal ideation and WCUS in adolescents with asthma has not been evaluated previously. To the best of our knowledge, our results are the first to show that sufficient WCUS duration (of [?]2hr) may play a protective role against suicidal ideation among adolescents with asthma. However, this finding does not mean that adolescents with asthma should sleep longer on the weekends than they do during weekdays. Nevertheless, it is important for adolescents with asthma to achieve sufficient sleep time during the weekend in the way that WCUS is an indicator of insufficient weekday sleep.

There are several methodological limitations in this study that must be noted. First, our results are subject to recall bias, because we used self-reported data collected via the internet. Studies using more objective assessments of sleep, such as polysomnography or actigraphy, are needed to avoid this limitation. Second, we only collected data at a single point in time by asking about typical sleep pattern over the preceding week. Weekend sleep duration may vary depending on the situation of the following week (e.g. school examination). Longitudinal studies are needed to clarify our findings, as asthma control and sleep patterns may change throughout the school year. Third, this study was cross-sectional in design; therefore, we were unable to establish a causal relationship between associated sleep factors and suicidal ideation in adolescents with asthma. Well-controlled prospective studies are necessary to confirm the results of the present study. Fourth, we did not analyze asthma management, treatment modalities, or asthma severity because such information was lack and only the lifelong diagnosis of asthma was evaluated in the survey. This omission may have led to the misclassification of asthma. In addition, despite adjusting for various potential confounders, the survey did not assess other significant factors that have been associated with poor sleep quality, such as OSA, snoring, nocturnal gastroesophageal reflux, internet or smartphone use time, and caffeine or drug use.

Despite these limitations, this study also has several strengths and important clinical implications for adolescents with both asthma and sleep problems. This was a nationwide, government-directed survey with a high response rate $(96.2 \%)$. To the best of our knowledge, our study included the largest number of 
participants among similar studies. A socioeconomically diverse sample with an equal proportion of middle school and high school students (400 schools each annually) were represented. All of the analyses in this study were based on sample weights and were adjusted for the complex sample design of the survey. Almost all participants had the same ethnic background, which minimizes other possible confounding factors. These study characteristics also mean that our results are generalizable to the overall Korean population. Most of all, this research was the first to identify associated sleep factors associated with suicidal ideation in adolescents with asthma. No prior study had evaluated the relationship between WCUS and asthma. The public health implications of our findings can be applied at the educational and practical levels. A recent study demonstrated that adolescents were able to successfully extend sleep duration with sleep hygiene education. ${ }^{33}$ This simple intervention would likely be beneficial for adolescents with asthma. ${ }^{12}$ From an educational perspective, strategic interventions to reduce academic stress on Korean adolescents should be seriously considered, such as a more late start to classes. Health care professionals, including pediatric allergists, require a greater awareness of the risk of mental health problems and suicidality in adolescents with asthma. This is particularly true in those with concurrent sleep problems. When treating adolescents with asthma, these patients require a holistic approach with a more accurate assessment of associated sleep factors related to suicidality.

\section{5 | CONCLUSION}

Although the conclusions of this study should be considered cautiously, our findings suggest that sleep dissatisfaction and short sleep duration are associated with an increased risk of suicidal ideation. However, long WCUS, used as a compensatory effort for insufficient weekday sleep, is associated with a decreased risk of suicidal ideation in Korean adolescents with asthma. Based on these results, more attention must be paid to asthmatic patients and their sleep duration and satisfaction in order to reduce the risk of suicidal ideation.

\section{ACKNOWLEDGMENTS}

We would like to thank eWorldEditing (www.eworldediting.com) for English language editing.

\section{CONFLICT OF INTERESTS}

All authors declared no conflict of interests.

\section{REFERENCES}

1. Lundback B, Backman H, Lotvall J, Ronmark E. Is asthma prevalence still increasing? Expert Rev Respir Med . 2016;10(1):39-51.

2. Duksal F, Becerir T, Ergin A, Akcay A, Guler N. The prevalence of asthma diagnosis and symptoms is still increasing in early adolescents in Turkey. Allergol Int . 2014;63(2):189-197.

3. Estrada RD, Ownby DR. Rural Asthma: Current Understanding of Prevalence, Patterns, and Interventions for Children and Adolescents. Curr Allergy Asthma Rep . 2017;17(6):37.

4. de Benedictis D, Bush A. Asthma in adolescence: Is there any news? Pediatr Pulmonol . 2017;52(1):129138.

5. Han CH, Chung JH. Association of asthma and sleep insufficiency among South Korean adolescents: analysis of web-based self-reported data from the Korean youth risk behavior web-based survey. $J$ Asthma . 2020;57(3):253-261.

6. Khan WH, Mohsenin V, D'Ambrosio CM. Sleep in asthma. Clin Chest Med . 2014;35(3):483-493.

7. Bakour C, O'Rourke K, Schwartz S, Wang W, Sappenfield W, Couluris M. Sleep duration, obesity, and asthma, in Florida adolescents: analysis of data from the Florida Youth Risk Behavior Survey (2009-2013).Sleep Breath . 2017;21(4):1039-1045.

8. Ferraz E, Borges MC, Vianna EO. Influence of nocturnal asthma on chronotype. J Asthma . 2008;45(10):911-915.

9. Han CH, Chung J. Late Chronotype is Associated with Adolescent Asthma: Assessment Using the Korean-Version MCTQ. Int J Environ Res Public Health . 2020;17(9).E3000. 
10. Vargas PA, Robles E. Asthma and allergy as risk factors for suicidal behavior among young adults. $J$ Am Coll Health . 2019;67(2):97-112.

11. Jensen ME, Gibson PG, Collins CE, Hilton JM, Latham-Smith F, Wood LG. Increased sleep latency and reduced sleep duration in children with asthma. Sleep Breath . 2013;17(1):281-287.

12. Meltzer LJ, Ullrich M, Szefler SJ. Sleep duration, sleep hygiene, and insomnia in adolescents with asthma. J Allergy Clin Immunol Pract . 2014;2(5):562-569.

13. Chugh IM, Khanna P, Shah A. Nocturnal symptoms and sleep disturbances in clinically stable asthmatic children. Asian Pac J Allergy Immunol . 2006;24(2-3):135-142.

14. Hwangbo Y, Kim WJ, Chu MK, Yun CH, Yang KI. Association between weekend catch-up sleep duration and hypertension in Korean adults. Sleep Med . 2013;14(6):549-554.

15. Koo DL, Yang KI, Kim JH, et al. Association between morningness-eveningness, sleep duration, weekend catch-up sleep and depression among Korean high-school students. J Sleep Res.2020:e13063.

16. Im HJ, Baek SH, Chu MK, et al. Association Between Weekend Catch-up Sleep and Lower Body Mass: Population-Based Study. Sleep . 2017;40(7).doi: 10.1093/sleep/zsx089.

17. Lv Y, Cai L, Zeng X, et al. Association between weekend catch-up sleep and executive functions in Chinese school-aged children. J Clin Sleep Med . 2020;13.doi: 10.5664/jcsm.8494.

18. Kang SG, Lee YJ, Kim SJ, et al. Weekend catch-up sleep is independently associated with suicide attempts and self-injury in Korean adolescents. Compr Psychiatry . 2014;55(2):319-325.

19. Lee SU, Park JI, Lee SJ, Oh IH, Choi JM, Oh CH. Changing trends in suicide rates in South Korea from 1993 to 2016: a descriptive study.BMJ Open 2018;28:8(9): e023144

20. Hirshkowitz M, Whiton K, Albert SM, et al. National Sleep Foundation's sleep time duration recommendations: methodology and results summary.Sleep Health . 2015;1(1):40-43.

21. Son SM, Park EJ, Cho YH, et al. Association Between Weekend Catch-Up Sleep and Metabolic Syndrome With Sleep Restriction in Korean Adults: A Cross-Sectional Study Using KNHANES. Diabetes Metab Syndr Obes . 2020;13:1465-1471.

22. Zhang J, Paksarian D, Lamers F, Hickie IB, He J, Merikangas KR. Sleep Patterns and Mental Health Correlates in US Adolescents. J Pediatr . 2017;182:137-143.

23. Yeo SC, Jos AM, Erwin C, et al. Associations of sleep duration on school nights with self-rated health, overweight, and depression symptoms in adolescents: Problems and possible solutions. Sleep Medicine . 2019:60:96-108.

24. Kavanagh J, Jackson DJ, Kent BD. Sleep and asthma. Curr Opin Pulm Med . 2018;24(6):569-573.

25. Barker E, Kolves K, De Leo D. The relationship between asthma and suicidal behaviours: a systematic literature review. Eur Respir J . 2015;46(1):96-106.

26. Jarosz M, Syed S, Błachut M, Badura Brzoza K. Emotional distress and quality of life in allergic diseases. Wiad Lek . 2020;73(2):370-373.

27. Chung JH, Kim SH, Lee YW. Suicidal ideation and suicide attempts among asthma. Ann Gen Psychiatry. 2016;15:35.

28. Zhang Y, Cheng J, Li Y, et al. Suicidality among patients with asthma: A systematic review and meta-analysis. J Affect Disord.2019;256:594-603.

29. Castro-Rodriguez JA, Brockmann PE, Marcus CL. Relation between asthma and sleep disordered breathing in children: is the association causal? Paediatr Respir Rev . 2017;22:72-75.

30. Trivedi M, ElMallah M, Bailey E, Kremer T, Rhein LM. Pediatric Obstructive Sleep Apnea and Asthma: Clinical Implications.Pediatr Ann . 2017;46(9):e332-e335.

31. Gutierrez MJ, Zhu J, Rodriguez-Martinez CE, Nino CL, Nino G. Nocturnal phenotypical features of obstructive sleep apnea (OSA) in asthmatic children. Pediatr Pulmonol . 2013;48(6):592-600.

32. Oh YH, Kim H, Kong M, Oh B, Moon JH. Association between weekend catch-up sleep and healthrelated quality of life of Korean adults.Medicine (Baltimore) . 2019;98(13):e14966.

33. Dewald-Kaufmann JF, Oort FJ, Meijer AM. The effects of sleep extension and sleep hygiene advice on sleep and depressive symptoms in adolescents: a randomized controlled trial. J Child Psychol Psychiatry . 2014;55(3):273-283.

\section{Hosted file}


Table1-4.docx available at https://authorea.com/users/334448/articles/460422-association-ofsleep-duration-and-weekend-catch-up-sleep-with-suicidal-ideation-among-adolescents-withasthma-secondary-data-analysis-based-on-a-nationwide-cross-sectional-study 